\title{
Labyrinthe
}

27 | 2007 (2)

La fin des disciplines?

\section{La « mluridisciplinarité » institutionnelle en France après mai 1968}

\section{Laurent Ferri}

Traducteur : Déborah Cohen, Grégoire Leménager et Arnault Skornicki

\section{OpenEdition \\ Journals}

Édition électronique

URL : https://journals.openedition.org/labyrinthe/2004

DOI : $10.4000 /$ labyrinthe.2004

ISSN : 1950-6031

Éditeur

Hermann

Édition imprimée

Date de publication : 4 juillet 2007

Pagination : 27-36

ISBN : 978-2-9526131-4-9

Référence électronique

Laurent Ferri, « La « mluridisciplinarité » institutionnelle en France après mai 1968 », Labyrinthe [En

ligne], 27 | 2007 (2), mis en ligne le 25 mars 2011, consulté le 28 avril 2022. URL : http://

journals.openedition.org/labyrinthe/2004; DOI : https://doi.org/10.4000/labyrinthe.2004

Propriété intellectuelle 


\title{
La fin des disciplines?
}

\section{LA «MLURIDISCIPLINARITÉ» INSTITUTIONNELLE EN FRANCE APRÈS MAI 1968}

\author{
Laurent FERRI \\ 1f66@cornell.edu
}

Cet article repose sur une idée très simple: les concepts ont une histoire et leur généalogie peut être dressée en détail. Comme celle de nos vies, cette histoire est une série de succès et d'échecs. Dans le cas d'«inter-» et de «pluri-disciplinarité», je voudrais montrer que ces concepts ont connu un succès tout particulier dans les vingt-cinq dernières années. Il est peu commun de voir un mot nouveau devenir tellement à la mode qu'il oriente rapidement jusqu'au discours législatif. Aujourd'hui, «interdisciplinarité », en particulier, est presque un sine qua non de tout discours sur l'Université.

Or, tout ceci s'est fait sur une très courte période: tandis qu'avant 1968 on ne trouvait dans la plupart des dictionnaires qu'une très brève notice, voire rien pour son acolyte «pluridisciplinarité», cette notion constitua un des trois piliers sur lesquels reposait la loi d'orientation de l'enseignement supérieur du 12 novembre 1968 (les deux autres étant «autonomie» et «participation»). L'article 6 stipule en effet que «les universités sont pluridisciplinaires et doivent associer autant que possible les arts et les lettres aux sciences et aux techniques ». Dans la foulée de l'adoption de la loi, des universités interdisciplinaires furent créées. Comment un changement d'une telle ampleur a-t-il pu se produire en si peu de temps?

Archiviste de formation et de goût, j'ai entrepris de sonder les documents de cette période afin de voir de quelle manière l'interdisciplinarité a trouvé sa place dans les nouvelles normes d'enseignement supérieur après Mai 68. Dans un pays aussi centralisé et culturellement unifié que la France des années 1960, toutes les décisions d'importance concernant l'université et la recherche partaient nécessairement du niveau ministériel. Les plus importantes émanaient de services dépendant directement 
du ministre, lui-même dépendant du Président. J'ai choisi de m'intéresser au cabinet d'Edgar Faure (1908-1988), nouveau ministre de l'Éducation nationale investi à la suite des élections de juillet 1968, largement remportées par le parti gaulliste. Il n'est pas question ici de retracer la longue carrière de cette figure clé des $\mathrm{IV}^{\mathrm{e}}$ et $\mathrm{V}^{\mathrm{e}}$ Républiques. Rappelons juste que cet habile politicien fut un «touche-à-tout» au profil très... pluridisciplinaire. Lauréat de la faculté de droit et diplômé des Langues $\mathrm{O}$, Edgar Faure devint un avocat et écrivain, n'hésitant pas à utiliser certaines affaires criminelles comme source d'inspiration pour une série de romans policiers. Mais Faure est aussi l'auteur de livres sur les finances publiques de l'Ancien Régime, sur Lénine, sur la Chine contemporaine ou sur la politique énergétique de la France. Ce fut avant tout un homme politique, mais d'une manière absolument éclectique et non conformiste. Après son ralliement à de Gaulle, Faure fut l'émissaire français dont la mission à Pékin en 1963 fut rapidement suivie de la reconnaissance par la France de la Chine communiste. Il en avait profité pour apprendre le chinois. En partie parce que, de 1966 à 1968, il avait eu quelques succès au ministère de l'Agriculture, il fut choisi comme ministre de l'Éducation nationale et le resta jusqu'en juin 1969. Véritable réformiste, ouvert d'esprit et passionné par la recherche du compromis, Faure promit en 1955 son fameux «changement dans la continuité». Il avait également un faible pour les «plans généraux» qui, si l'expression a des relents de Gosplan soviétique, n'en étaient pas moins caractéristiques de la politique menée à l'époque.

J'ai donc consulté les cinq cartons du Fonds Edgar Faure enregistrés aux Archives nationales sous la cote 505 AP. Traquer les occurrences de «pluridisciplinarité» et d' 'interdisciplinarité» dans des documents administratifs peut rendre aussi optimiste que chercher une aiguille dans une botte de foin. De fait, la lecture des instruments de recherche fut décevante: lorsque le mot «discipline» apparaissait, il faisait toujours référence à la nécessité de «remettre les chose en ordre» après les émeutes estudiantines. Toutefois, mes recherches finirent par payer: les termes «pluridisciplinarité» et «interdisciplinarité» (ainsi que des synonymes) sont utilisés de manière fréquente dans les notes au ministre, les rapports, les projets de loi et les interviews. À dire vrai, même après le vote de la loi de novembre 1968, certaines distinctions demeurèrent pour le moins floues. Un mémorandum envoyé à de Gaulle en mars 1969 révèle de manière involontairement comique la perplexité des conseillers du ministre: 
Les réflexions que nous poursuivons actuellement en vue de déterminer ce que sont exactement l'interdisciplinarité, la pluridisciplinarité, la multidisciplinarité, l'instrumentalité réciproque des disciplines ou la transposition des modèles disciplinaires nous amènent à rencontrer les thèmes et les préoccupations qui sont aujourd'hui celles de l'enseignement supérieur dans les pays avancés (p. 45).

Tout ceci n'est pas sans évoquer les interminables (et fructueuses) séances de typologie sur ces questions au sein de Labyrinthe! En raison d'une faute de frappe, je suis même tombé sur une délicieuse «mluridisciplinarité » :

L'un des principaux objectifs de la loi d'orientation et de réforme de l'Université réside dans l'effort vers la généralisation de l'enseignement mluri-disciplinaire (ibid.).

Il convient de rappeler brièvement ce qu'était la «philosophie de la réforme », puis de donner quelques exemples de la manière dont elle fut conduite; enfin, nous tenterons de prendre la mesure des réalisations, c'est-à-dire de l'impact de la pluridisciplinarité et de l'interdisciplinarité sur des universités très compartimentées.

Sur ce point, deux précautions s'imposent. Tout d'abord, je m'appuie sur des archives qui ne donnent qu'un premier aperçu de l'activité ministérielle sur une période de deux ans (1968-1969) et non une vue précise de la mise en œuvre concrète de la réforme au jour le jour. Deuxièmement, je sais que l'historien court toujours le risque de manquer la vision d'ensemble, à trop se concentrer sur son sujet. En l'occurrence, il serait assez ridicule de prétendre que la «pluridisciplinarité» fut, pour qui que ce soit, l'enjeu central de 1968. Même pour les étudiants et les quelques enseignants qui s'intéressaient à la question, ce ne fut jamais qu'un horizon lointain, ou un enjeu très marginal, alors que beaucoup rêvaient de changer le monde. Je pense néanmoins que nous aurions tort de ne voir dans la «pluridisciplinarité» qu'une sorte d'engouement passager ou de marotte technocratique. La pluridisciplinarité fut un enjeu réel que ni les réformistes ni les révolutionnaires ne pouvaient ignorer ou négliger.

Avant 1968, l'Université - notez le singulier, car cette institution était fort monolithique - était tout sauf pluridisciplinaire. Si l'on met de côté les facultés de droit et de médecine, elle était avant tout destinée à 
former les futurs docteurs et enseignants spécialisés dans une discipline. Cette réduction de la fonction scientifique de l'Université à la formation professionnelle, en vue de parcours très «balisés», avait pour conséquence imparable que la recherche ne pouvait pénétrer cet univers hypercompartimenté. Dans une étude magistrale, contemporaine des «événements de mai», Alain Touraine consacrait un chapitre à «La crise de l'Université» où l'on pouvait lire ceci:

Un helléniste n'aura pas connaissance des travaux de l'ethnologue; un historien ignorera à peu près tout de la sociologie et de l'économie moderne; le sociologue, dont la formation est plus diversifiée, ignorera tout des méthodes de la biologie, sans parler de son ignorance officialisée par les récentes réformes, de l'histoire des sociétés qu'il étudie. Tous n'acceptent pas cette professionnalisation et cherchent alors hors de la formation universitaire une ouverture d'esprit, une diversité d'informations que la faculté leur refuse. Les revues littéraires et politiques, les hebdomadaires de grande information, la télévision ou mieux encore des groupements d'inspiration politique ou religieuse, complètent la formation professionnelle donnée par l'Université, ce qui crée une rupture profonde entre les éléments de l'éducation qui devraient être unis ${ }^{1}$.

La réforme Fouchet-Aigrain, introduite en 1966, avait certes tenté de lever les barrières entre les facultés, en autorisant les étudiants à suivre des études interdisciplinaires. Mais l'effet obtenu fut exactement inverse. La séparation entre les facultés fut renforcée, et les étudiants devinrent plus liés à une spécialité qu'auparavant. Quelques savants distingués comme Georges Gusdorf, professeur de philosophie à Strasbourg de 1948 à 1976, réagit contre l'étroite spécialisation professionnelle de l'Université, en attirant l'attention sur les sociétés traditionnelles où l'apprentissage sur le tas constituait le noyau de la formation professionnelle. Si l'on appliquait ce modèle en France, l'Université pourrait se concentrer sur les grandes idées et la «culture générale». Comme Touraine le souligne:

1. Alain Touraine, Le Mouvement de Mai ou le communisme utopique, Paris, Le Seuil, coll. «Politique», 1972, p. 88. 
Le paradoxe est que l'Université, au moment même où elle affirme l'éminence et les droits de la pensée désintéressée, est la plus fidèle à l'opposition entre la technique servile et la pensée noble ${ }^{1}$.

Une telle dichotomie convenait davantage à la Grèce ancienne qu'à la France moderne... En outre, Gusdorf ignorait l'aspect social du problème, sur lequel j'aimerais insister.

Alors que la concentration sur la formation professionnelle écartait de l'Université la majeure partie des élites socioculturelles, qui continuaient de lui préférer les grandes écoles, à partir des années 1960 les «facs» commencèrent à accueillir un nombre toujours accru d'étudiants. C'était une tendance positive en termes de démocratisation et d'augmentation des effectifs du corps enseignant. Avant les années 1960, la plupart des universités de province ne comptaient généralement qu'un professeur par discipline, ce qui limitait automatiquement l'interdisciplinarité. Cependant, la pression du nombre aggrava les problèmes de l'Université, confrontée à l'arrivée en masse d'étudiants sans cultural background. Pour la plupart d'entre eux, les études supérieures ne constituaient qu'un bref intermède culturel; «ils ne quittaient l'université qu'avec des connaissances disparates, et pour trouver des emplois médiocres ${ }^{2} \gg$, voire pour se retrouver au chômage - c'était le cas de nombreux diplômés en sociologie et en psychologie. De ce point de vue, la «pluridisciplinarité» n'était pas seulement le nouveau discours de la méthode élaboré rue de Grenelle: c'était également une question de débouchés et de subsistance.

Edgar Faure envisageait le décloisonnement disciplinaire comme un moyen de réformer l'enseignement supérieur afin qu'il soit mieux adapté aux besoins de la société. La pensée technocratique de l'époque plaidait pour plus de «fluidité», une notion incluant à la fois la flexibilité du marché du travail et l'ouverture d'esprit d'une société française qui manquait cruellement de mobilité et de mixité sociales ${ }^{3}$. Dans les rapports du cabinet d'Edgar Faure, le nouveau produit de l'université du futur s'appelle «l'honnête homme du XXI ${ }^{\mathrm{e}}$ siècle» (note adressée à de Gaulle, mars 1969), ou «l'homme pluridimensionnel» (note sur

1. Idem, p. 78.

2. Michelle Paterson, «French University Reform: Renaissance or Restoration?», dans Comparative Education Review, 1972, p. 285.

3. Le sociologue Michel Crozier allait bientôt en faire le thème de son best-seller La Société bloquée, Paris, Le Seuil, 1971. 
Vincennes, s. d.) $)^{1}$. Lors d'un discours prononcé devant les inspecteurs généraux de l'Éducation nationale, le ministre lança l'expression «Cgentleman» (en anglais) pour décrire les lycéens bien équipés pour rejoindre l'Université:

Ce n'est ni un bourreau de travail, ni bien sur un cancre, mais un individu capable de se tenir, avec élégance, dans la moyenne².

Le «C-gentleman» serait apte à exercer les emplois les plus qualifiés dans la prétendue «société postindustrielle ${ }^{3}$ ». La notion d'un «emploi pour la vie» appartenant au passé, l'idée était que seule la "pluridisciplinarité» permettrait à chacun "l'adaptation ultérieure à un autre emploi que celui pour lequel on avait été spécifiquement formé». Faure croyait dans la démocratisation de l'enseignement supérieur comme faisant partie intégrante d'une démocratisation de l'ensemble de la société; celle-ci devait, selon lui, être dominée par une classe moyenne instruite et dynamique, non par une aristocratie. Il ne manquait pas une occasion de railler ses opposants élitistes: par exemple, le président de la très conservatrice Société des agrégés ayant déclaré dans Le Figaro que «l'accès à toutes les facultés devrait être réservé aux seuls bacheliers convenables», Faure griffonna en marge de l'article: «Qu'est-ce qu'une femme convenable?»

Mais comment la «pluridisciplinarité» fut-elle mise en œuvre et favorisée? Une mesure importante fut le démantèlement des 115 universités au profit de la création de 720 nouvelles «unités d'enseignement et de recherche» (UER). La portée interdisciplinaire de la réforme fut limitée du fait que la plupart de ces UER étaient formées à partir d'une section particulière de l'Université, ou encore sur la base d'affinités plus politiques qu'intellectuelles. Aucune ligne de conduite ne fut assignée par le ministère, et le résultat fut que, au lieu d'avoir 115 unités, les universités furent désormais composées de 720 plus petites unités tout aussi cloisonnées ${ }^{4}$.

\footnotetext{
1. Ce renvoi discret et superficiel à «L'homme unidimensionnel» d'Herbert Marcuse (1964) témoigne de ce que les technocrates tentaient de suivre les nouvelles «modes» intellectuelles.

2. Dans le système de notation américain traditionnel, $\mathrm{C}$ serait l'équivalent de la mention passable. Politicien radical, Faure se souvenait du «Français moyen» d'Édouard Herriot.

3. L'expression ne fut inventée qu'en 1973, mais l'idée que la France et les autres «pays avancés» allaient vers des «sociétés de services» faisait déjà partie de la vulgate technocratique.

4. Michelle Paterson, «French University...», art. cit., p. 289.
} 
Une autre idée fut de fédérer les disciplines et les enseignements autour d'un thème commun, ou autour de nouvelles «disciplines »: dans les papiers de Faure, on trouve par exemple une note enthousiaste, datée du 2 décembre 1969, sur «l'enseignement des sciences de l'environnement à l'université de (sic) Wisconsin». En principe, cependant, la «pluridisciplinarité » signifiait simplement un plus grand choix de disciplines proposées simultanément, mais sans association ni croisement. En outre, la spécialisation restait l'objectif. Par exemple, dans un entretien accordé à la revue économique Le Dirigeant, Faure déclara:

L'enseignement des affaires doit se poursuivre dans le premier cycle de l'enseignement supérieur par l'association pluridisciplinaire de disciplines telles que la science économique, les langues vivantes, le droit, la sociologie, la science politique, les mathématiques appliquées, l'informatique. Il doit se spécialiser dans le second cycle à partir de ces bases communes, tandis que le troisième cycle est réservé à la recherche.

Un troisième élément de la réforme fut la création, dans l'académie de Paris, de nouvelles universités expérimentales «interdisciplinaires » (et non «pluridisciplinaires»). Mais pas davantage ici qu'ailleurs, encore, l'importance accordée à la pluri- et à l'interdisciplinarité sur la nouvelle scène universitaire n'était clairement établie. Une note récapitulative du 29 août 1968 le met en évidence. Elle indique que «Dauphine» proposera «des séminaires de recherche interdisciplinaires». À «Montrouge», lit-on, «l'enseignement sera donné par des professeurs des facultés de sciences et de médecine appelés à travailler ensemble», tandis que le premier cycle d'études de médecine proposera «un large éventail d'options, des mathématiques à la physique, de la psychologie aux langues vivantes ». En définitive, c'est seulement à Vincennes qu'une véritable interdisciplinarité fut mise en œuvre «à tous les niveaux», et qu'elle fut elle-même un objet de recherche, en particulier au sein du «Centre de recherches en méthodologie pluridisciplinaire». Dans un geste très audacieux, Faure donna aux academics non conformistes et révolutionnaires un espace de liberté. Sous la pression du syndicat Snes-Sup, un célèbre «groupe cooptant» d'environ trente membres (Hélène Cixous étant de loin la plus influente) eut la prérogative insolite de recruter le reste du corps enseignant. En ce qui concerne le cadre pédagogique adopté, Guy Berger ${ }^{1}$ a récemment rappelé :

1. Voir note p. 34 p. 


\section{Labyrinthe, $n^{\circ} 27$}

L'université américaine servit de modèle pour Vincennes. Las Vergnas ${ }^{1}$ a été désigné par Edgar Faure comme chargé de mission, et lui-même a désigné un certain nombre de maîtres-assistants, généralement américanistes. Le modèle est une université américaine, où l'organisation pédagogique prend la forme de crédits, avec une grande liberté de choix. Ce qui entraîne une pluridisciplinarité de fait, avec chaque fois que possible des travaux de petits groupes, l'introduction de technologies audio-visuelles, mais aussi l'idée qu'une langue vivante et l'informatique, dont on commence à parler, seront obligatoires. C'est Pierre Doumergue qui est porteur de ce projet. En liaison/contradiction avec ce projet, il y a un projet intellectuel, qui donne une place essentielle à des disciplines ou à des conceptions des disciplines reconnues, mais qui sont complètement ou partiellement exclues du monde universitaire. Hélène Cixous a une place majeure dans ce projet. Quelles disciplines? La psychanalyse, sous sa forme lacanienne, mais qui sera essentiellement représentée par Serge Leclaire - un des cooptants. L'urbanisme, qui jusque-là est une vague filière soit des études d'architecture, soit de certaines écoles d'ingénieurs. Mais aussi des conceptions marginalisées de disciplines classiques: la théorie du texte, dont Jean-Pierre Richard est une figure emblématique, la sociologie de Bourdieu qui jusque-là est non reconnue ou dispersée dans plusieurs universités².

En termes de pluri-/interdisciplinarité, il est remarquable les «sciences dures» aient eu leur place dans le projet initial de Vincennes, mais que cette composante ait été abandonnée.

Finalement, l'interdisciplinarité a-t-elle "percé» en 1968 ? Je serais tenté de reprendre une expression chère à Edgar Faure, «le changement dans la continuité». Inter- et pluridisciplinarité n'ont pas connu un développement spectaculaire, et il est difficile sur ce point de ne pas blâmer le ministère pour sa conception verticale de la politique, et pour certaines incohérences. En voici une parmi d'autres: tandis que l'interdisciplina-

\footnotetext{
1. Guy Berger est professeur émérite à Paris-8, et «accessoirement» l'ex-époux d'Hélène Cixous. Sur le rôle de cette dernière, on peut désormais consulter un fonds d'archives à la Bibliothèque nationale de France.

2. Directeur de l'Institut d'anglais de la Sorbonne jusqu'en mai 1968, Raymond Las Vergnas avait été élu, durant «les événements de Mai», doyen de la vénérable université, alors en plein chambardement. Il est probable que, sans cet honnête homme, «assisté» de personnalités aussi diverses que le vicedoyen Jean-Baptiste Duroselle et Cixous, Vincennes n'aurait pu se faire.

3. Entretien accordé à Maurice Courtois pour le Magazine de l'université Paris 8 , juin 2002, accessible en ligne, URL http://www.ipt.univ-paris8.fr/hist/Interview_Berger.htm [page consultée le 28 mai].
} 
rité était officiellement encouragée dans les nouvelles universités «expérimentales» en région parisienne (et expérimentale, la démarche le fut indéniablement dans une université comme celle de Vincennes), ce qui semble avoir été prescrit pour les petites et moyennes universités de province fut au contraire un renforcement de la spécialisation, pour diverses raisons, notamment afin de les adapter davantage à leur environnement économique régional. Par exemple, Besançon (un cas bien documenté, car Edgar Faure disposait d'un importante assise politique en FrancheComté1) devait, selon le cabinet, consolider ses programmes scientifiques en optique et en métrologie, parce que les industries régionales dominantes y étaient - pour peu de temps encore - l'horlogerie et la fabrication de lunettes.

Mais plutôt que sur cette observation, je voudrais conclure sur une autre idée. Pratiquer la pluri- et l'interdisciplinarité ne dépend pas en tout premier lieu d'un cadre institutionnel, des règles avec lesquelles les étudiants et les chercheurs doivent vivre, même si l'autonomie, la participation, un financement adapté, des statuts idoines sont extrêmement importants. La pluridisciplinarité reste pour l'essentiel une question d'initiatives individuelles et de dynamiques inter-individuelles. La seule occurrence de cette notion dans les «papiers Faure» se trouve - de manière tout sauf incidente - dans une note sur les universités américaines, datée du 15 octobre 1968 :

Les professeurs ont tous des bureaux où ils sont pratiquement huit heures par jour, ce qui facilite les rencontres avec les occupants des bureaux voisins, sans parler des contacts téléphoniques. Ils prennent leurs déjeuners à la cafétéria ou dans un club de professeurs, ce qui donne encore des facilités pour les rencontres. Mais, indépendamment de ces facilités matérielles, il y a toutes les habitudes et tous les comportements psychologiques et sociologiques de la société américaine qui font que on (sic) a beaucoup moins tendance qu'en France à se replier sur soi-même et, qu'au contraire, on parle plus volontiers aux collègues de ses recherches, de ses enseignements et de toutes sortes d'autres sujets. Il faut dire aussi que le professeur américain a

\footnotetext{
1. Il fut en effet, successivement ou simultanément, député radical-socialiste du Jura (1946-1958), maire de Port-Lesney (1947-1970), président du Comité d'expansion économique de Franche-Comté et du Territoire de Belfort (1951) puis de la Commission de développement économique régional de Franche-Comté (1964-1973), conseiller général du canton de Pontarlier (1967-1979), maire de Pontarlier (1971-1978), président du Conseil régional de Franche-Comté (1974-1988).
} 


\section{Labyrinthe, $n^{\circ} 27$}

probablement moins d'ambitions personnelles que le professeur français, et trouve parfaitement satisfaisant d'avoir à participer à titre anonyme à une recherche collective.

Il est donc évident que les structures de recherches, les bibliothèques, les archives, les revues, les éditeurs, et, oui, même les cafétérias sont des atouts essentiels au développement de l'interdisciplinarité. Pourtant, je voudrais soutenir que le plus important reste que des gens aient le désir de travailler ensemble, qu'ils souhaitent partager un territoire: j'emploie ce terme dans son sens intellectuel, pour désigner des champs du savoir, mais aussi dans un sens très pratique. Ne pas avoir l'esprit «territorial» est crucial, mais il y a plus. Je viens de lire un recueil de témoignages d'historiens américains spécialistes de la France, et j'ai été frappé par la remarque suivante:

Norbert Elias avait retenu de son exil à Paris en 1933 et 1934 cette impression: «J'aimais la France, j'aimais Paris, c'est pourquoi j'étais si malheureux qu'aucun collègue ne m'invite chez lui. Ce n'était pas quelque chose qu'on faisait.» De la lecture des historiens américains et canadiens ayant participé à ce volume, il ressort clairement que cette mauvaise habitude constitue toujours la norme ${ }^{1}$.

Considérons notre petit atelier de recherche para-institutionnel, Labyrinthe: où avons-nous expérimenté, de la manière la plus vivante et vibrante qui soit, les exigences constitutives et souvent irréconciliables de l'interdisciplinarité? Dans les salons-salles à manger de nos sympathiques studios parisiens, ou dans le brouhaha chaleureux des cafés. Précisément, parce que j'ai la conviction que l'on peut très difficilement pratiquer l'interdisciplinarité SEUL, et sans éprouver l'appartenance à une communauté de chercheurs, je voudrais terminer par ce mot que Jacques Derrida utilisait si souvent pour décrire ce qui devrait être notre premier mouvement quand nous nous tenons sur une frontière, dans la surprise initiale du contact avec un «autre», un étranger: hospitalité.

Traduit de l'anglais par Déborah Cohen, Grégoire Leménager et Arnault Skornicki

\footnotetext{
1. Roger Chartier, postface à l'ouvrage dirigé par Laura Lee Down et Stéphane Gerson, Why France? American Historian reflect on an Enduring Fascination, Ithaca et Londres, Cornell University Press, 2007, p. 228.
} 